the first HIV-1 fusion inhibitory peptide-based anti-HIV drug. However, its clinical application is limited because of its low potency and low genetic barrier to resistance. Furthermore, its mechanism of action is still elusive. Therefore, it is essential to define T20's mechanism of action, based on which a new analogous peptide with improved antiviral activity against divergent HIV-1 strains, including those resistant to T20.

Disclosure No significant relationships.

\section{P850 GENDER IDENTIFY AND SELF-REPORTED HPV VACCINATION AMONG YOUTH LIVING WITH OR AT HIGH-RISK FOR HIV}

'Elizabeth Arnold*, ${ }^{2}$ Dallas Swendeman, ${ }^{2}$ Cameron Goldbeck, 'Kate Bridges, ${ }^{3}$ Sue Ellen Abdalian, ${ }^{4}$ Jeffrey Klausner. ${ }^{1}$ UT Southwestern Medical Center, Dallas, USA; ${ }^{2}$ University of California Los Angeles, Los Angeles, USA; ${ }^{3}$ Tulane University, New Orleans, USA; ${ }^{4}$ UCLA David Geffen School of Medicine and Fielding School of Public Health, Epidemiology, Los Angeles, USA

\subsection{6/sextrans-2019-sti.892}

Background Human Papillomavirus (HPV) is common and is linked to cancer in both males and females. Efforts are underway to increase uptake of the vaccine (Gardasil) that is now recommended for youth and adults up to age 45 . However, little is known about the role of gender identify on vaccination, particularly among youth at increased risk for contracting and spreading the virus.

Methods We analyzed data $(\mathrm{N}=1070)$ from a longitudinal study of high-risk HIV negative youth and youth living with HIV aged 12-24. Data were collected in Los Angeles, CA and New Orleans, LA from clinic and community settings. We examined self-reports of HPV vaccination as well as predictors of vaccination.

Results Cis-gender females (CGFs) had the highest proportion of youth reporting vaccination $(52.7 \%)$ followed by transgender youth (45.9\%), gender non-conforming youth (44.2\%), and MSM (39.0\%). Cis-gendered, heterosexual males (CGHMs) reported the lowest proportion (32.9\%). However, $18.8 \%$ of youth did not know if they had received the vaccine. In our multivariate logistic model examining predictors of vaccination, gender identity was only significant for CGHMs. Other significant variables included age, some education higher than a high school diploma or completing higher education compared to less than high school, ever being tested for Hepatitis C, currently having a healthcare provider, always using condoms, and Latino or other race/ethnicity compared to African-American.

Conclusion Results suggest that despite national efforts to increase the uptake of the vaccine, many youth at high-risk for HPV have not received it or did not know if they had. CGFs appear to have the highest uptake, but CGMs are a group for whom increased efforts targeting vaccination are needed. Demographic and health care factors may also impact vaccination rates. Research is needed on barriers to vaccination and new interventions that go beyond education and promotion such as policy initiatives.

Disclosure No significant relationships.
P851 PERFORMANCE DISK DIFFUSION METHOD IN IDENTIFYING GONOCOCCAL RESISTANCE

${ }^{1}$ Wenqi Xu*, ${ }^{1}$ Jingwei Liu, ${ }^{1}$ Xiaoyu Zhu, ${ }^{2}$ Yueping Yin. ${ }^{1}$ National Center for STD Control, Chinese Center for Disease Control and Prevention, Chinese Academy of Medical Sciences and Peking Union Medical College, Nanjing, China; ${ }^{2}$ National Center for STD Control, Chinese Center for Disease Control and Prevention, Nanjing, China; Institute of Dermatology, Chinese Academy of Medical Sciences and Peking Union Medical College, Nanjing, China

\subsection{6/sextrans-2019-sti.893}

Background Because of their simple and cost-effective characteristics, many products of disk diffusion tests are commanly used by microbiological laboratories and hospitals world-wide to determine the gonococcal susceptibility and guide clinical treatments. To evaluate the reliability of routine detection of antimicrobial susceptibility of Neisseria gonorrhoeae by disk diffusion method, six kinds of discs targeted at the specific antibiotics (penicillin, ceftriaxone, cefixime, tetracycline, ciprofloxacin and spectinomycin) from two domestic and one foreign manufacturer were included into the evaluation using agar dilution method as a reference method to validate the concordance between the two methods.

Methods According to CLSI 2018 (M100, 28 ${ }^{\text {th }}$ ed) standard, the antimicrobial sensitivity results of more than 100 clinical isolates and 6 reference strains were interpreted. The coincidence rates of the two methods were analyzed by calculating four indicators: categorical agreement (CA), very major errors (VME), major errors (ME), minus errors (MIE). In addition, the correlation between agar dilution and disk diffusion methods were analyzed by using breakpoints established by linear least-squares regression.

Results The results showed that the MIC values of the 6 antibiotics on reference strains determined by the two methods were within the reference ranges. No very major or major discrepancies were detected in the 6 antibiotics. The performance of disk diffusion method to detect gonococcal susceptibility indicted that 1) the coincidence rates in spectinomycin and ciprofloxacin were high $(\mathrm{CA} \geq 98 \%) ; 2$ ) disk dilution method could miss some penicillin-resistant strains; (3) the coincidence rate of ceftriaxone and cefixime was low $(\mathrm{CA}<80 \%)$, in which agar dilution method is needed to confirm the detection of non-sensitive strains.

Conclusion The domestic products for ciprofloxacin, tetracycline, spectinomycin, cefixime and ceftriaxone were as satisfactory as foreign ones but the domestic for penicillin was relatively poor in quality.

Disclosure No significant relationships.

\section{P852 EVALUATION OF PLEXPCR ${ }^{\varpi}$ VHS FOR DIAGNOSIS OF GENITAL LESIONS}

${ }^{1}$ Litty Tan*, ${ }^{1}$ Peter Njuguna, ${ }^{1}$ Simon Erskine, ${ }^{2}$ Janet Towns, ${ }^{2}$ Marcus Chen, ${ }^{3} J u l i a n$ Druce, ${ }^{3}$ Franca Azzato, ${ }^{3}$ Thomas Tran, ${ }^{1}$ Elisa Mokany. 'SpeeDx Pty Ltd., Sydney, Australia; ${ }^{2}$ Melbourne Sexual Health Centre, Melbourne, Australia; ${ }^{3}$ Victorian Infectious Diseases Reference Laboratory, Melbourne, Australia

10.1136/sextrans-2019-sti.894

Background Genital lesions can be caused by herpes simplex virus types 1 and 2 (HSV-1 and HSV-2), varicella zoster virus (VZV) and Treponema pallidum (TP). Clinical presentations 